\title{
Abdominal pain manifesting intussusception in a rare adult Henoch-Schönlein purpura
}

\section{Early detection is key to treating the complication, urged researchers}

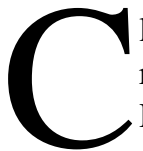

linicians in Poland have recently encountered a rare instance of children's disease Henoch-Schönlein purpura (HSP) affecting an adult - a case made even more unique with the occurrence of intussusception, or the "telescoping" of the intestines, itself a very uncommon HSP complication.

Clinician Natalia Zdanowska and her research team members from the Department of Dermatology at the University of Warmia and Mazury in Olsztyn, Poland, detailed their observation of the case in a report published by the Journal of Surgical Dermatology. "We decided to describe the case because of the relatively rare occurrence of Henoch-Schönlein purpura in adults," the authors said.
HSP is an acute systemic vascular disease that causes small blood vessels particularly in the skin, intestines, kidneys and joints to become inflamed and subsequently bleed. HSP's hallmark clinical feature is rash, or purpura, in the form of red-purple bruises caused by small bleeding vessels near the skin surface and covering all over the lower limbs and buttocks; however, other typical symptoms of HSP may also include bloody stools, joint pain and abdominal pain.

While much of HSP's origin is still unclear to modern medicine, it is already known to be overwhelmingly a childhood ailment and a very common type of vasculitis (inflammation of the blood vessels) in children: with 20 diagnosed cases per 100,000 children annually, HSP is the highest incident rate of vasculitis in pediatric patients. Most HSP cases occur in patients between the ages of 2

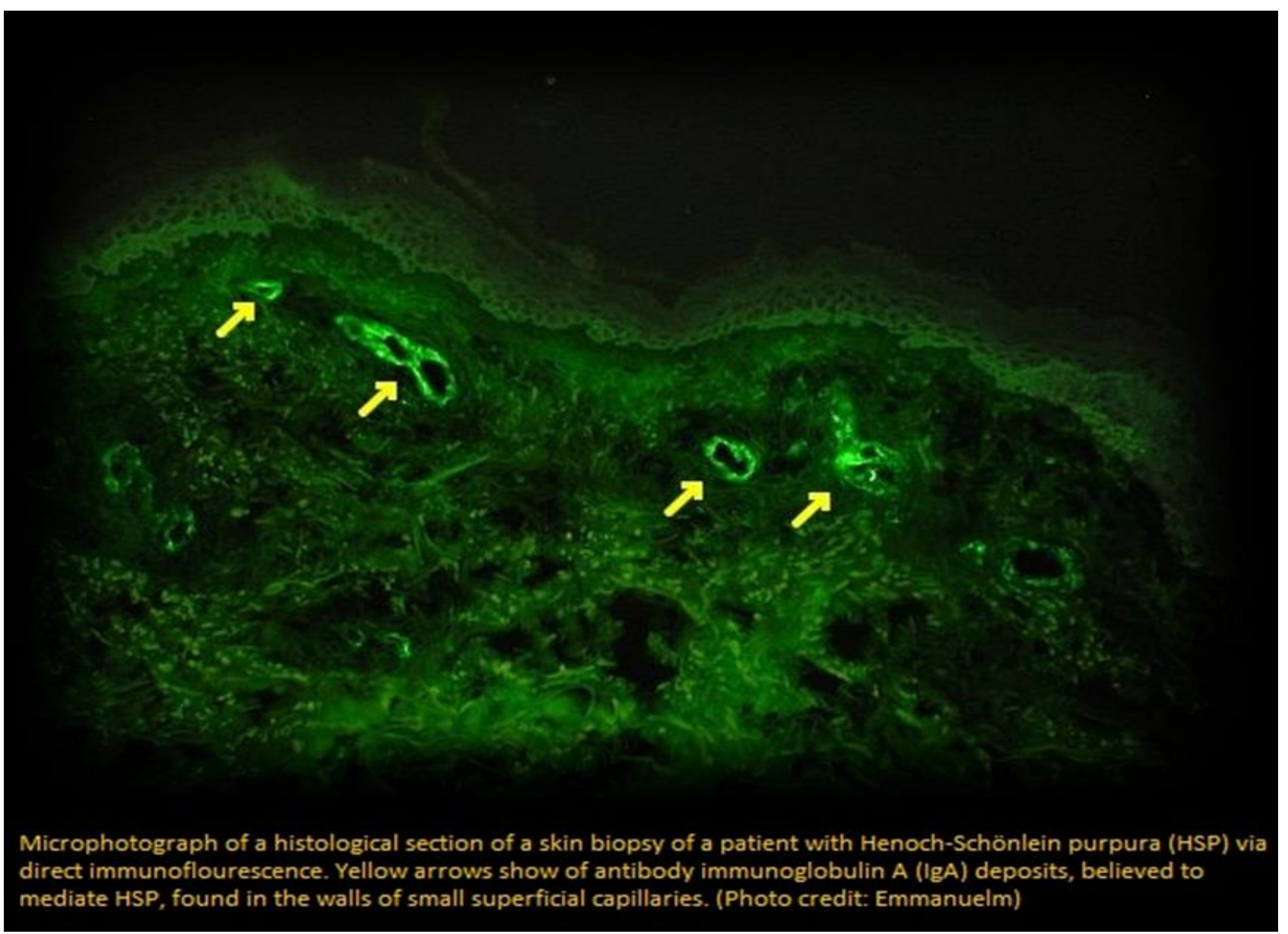


and 11, according to Mayo Clinic, with half of the affected patients being under the age of six, and $90 \%$ under the age of ten, occurring nearly twice as often in boys as in girls, and affecting Caucasian and Asian children more than others.

Rarely, however, do HSP afflict adults. When it does, according to the researchers, "the course of HSP in adults is usually more severe." While complications from HSP - which include bowel bleeding, bowel intussusception, and kidney failure - are typically rare, some adults HSP patients "require careful monitoring and surgical intervention because misdiagnosis can be life threatening," the researchers cautioned.

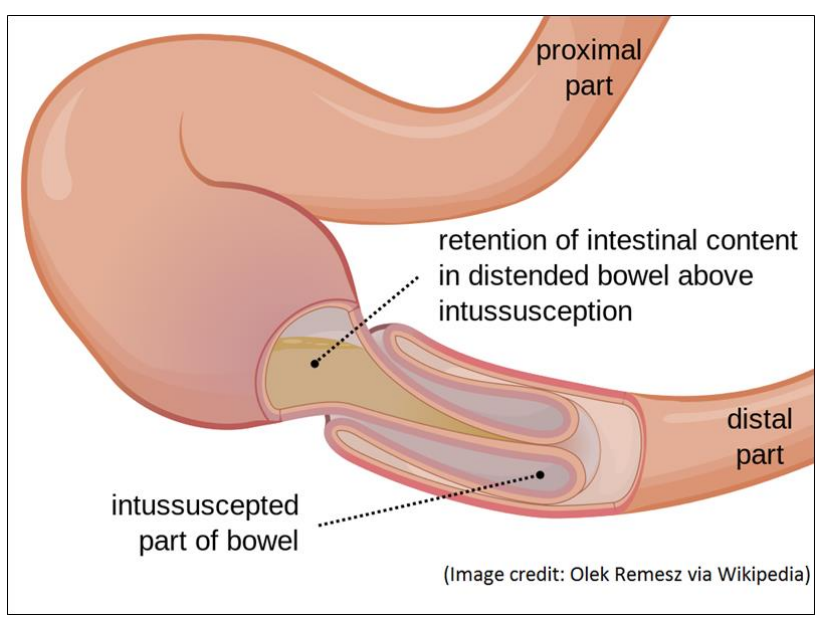

The report showcased a 23-year-old male patient who, in the course of HSP, developed intussusception-the folding of a section of the intestine into an adjacent part, akin to the folding of a telescope - of the jejunal (i.e., the second) segment of his intestine.

"Intussusception, along with severe complications of HSP, very seldom occurs in adults," wrote the researchers. Intussusception affects mostly infants under the age of 2, and adult cases of intussusception represent only $1 \%$ of all bowel obstructions. Even rarer are the adult cases of intussusception presenting as a complication arising from HSP. The authors' own review of medical literatures unearthed just two studies which altogether evaluated over 300 adults diagnosed with HSP but noted no occurrence of intussusceptions. A separate review revealed only two cases of colocolic intussusception associated with HSP in adults was found in Japan, Zdanowska and her team said.
Modern medical experts still do not know what exactly causes intussusception. HSP notwithstanding, intussusception is a serious medical disorder that causes bowel obstruction, which blocks food or fluid from passing through the stomach and cuts off blood supply to the affected part of the intestine. In turn, this could lead to the rupture in the bowel, along with an infection and death of bowel tissue. If left untreated, intussusception could turn fatal in mere 2-5 days.

In the case of the adult HSP patient featured in the report, after he was treated for skin lesions on his upper and lower limbs and for a mild upper respiratory infection, the patient was admitted to the hospital due to symptoms of acute abdomen which was diagnosed as jejunal intussusception, and he was urgently operated upon.

"During the operation, $20 \mathrm{~cm}$ resection of the small intestine was performed," Zdanowska and her fellow authors reported. Histopathology test showed "necrosis of the walls of the small intestine," confirming death of tissue due to the lack of blood flow, the researchers described. Post-surgery, the patient was treated with intravenous steroid medication dexamethasone and with topical application, and was soon discharged in good general condition with no lesions recurring.

Hence, the prognosis of intussusception is good, even in the case of HSP, as long as diagnosis is made early and the treatment is quickly administered. Otherwise, a prolapsed intussusception could aggravate other complications that could quickly lead to death. Unlike children with intussusception, adults rarely suffer from the occurrence of abdominal pain. When it does manifest, however, the authors recommend that HSP patients are examined for intussusception: "Therefore, in each patient who suffers from HSP with abdominal pain, this symptom should be considered," they concluded.

The case was reported by Natalia Zdanowska, Agnieszka Owczarczyk-Saczonek and Waldemar Placek. It is published in this issue of JSD (pages 11-13). 